\title{
A Component Based Approach Improves Classification of Discrete Facial Expressions Over a Holistic Approach
}

\author{
Kenny Hong, and Stephan K. Chalup, Senior Member, IEEE and Robert A.R. King
}

\begin{abstract}
Current approaches to facial expression classification employ a variety of expression classes and different preprocessing steps, making comparison of results difficult. To outline the effects of these variations we explore several image and action preprocessing steps, using the discrete expressions: happy, sad, surprised, fearful, angry, disgusted and neutral; with a dataset aligned and normalised by our proposed face model. Each of the preprocessing steps is organised across four prominent approaches: holistic, holistic action, component and component action. These are compared using a modified multiclass Support Vector Machine (SVM) that uses pairwise adaptive model parameters. We illustrate that including the neutral expression as part of the study has a noticeable impact, and suggest that it should be used in future research in this area. We also show that results can be improved through innovative use of image and action preprocessing steps. Our best correct classification rate was $98.33 \%$ using 10 -fold cross validation and a component action approach.
\end{abstract}

\section{INTRODUCTION}

Facial expression classification has gained popularity because of its application potential towards human computer interaction (HCI), where the ability to automate the capture of emotions, personality traits and cognitive intentions from facial expressions [1] was a significant advancement [2]. Current approaches to facial expression classification employ a variety of expression classes and different preprocessing steps, which makes comparison of results between studies difficult ([3], [4], [5]).

Happy, sad, surprised, fearful, angry and disgusted are the typical expression classes studied ([6], [7], [8]), and neutral is used as a reference image in a dynamic analysis (aka holistic action/component action) ([9], [10], [11], [12], [13], [14]) and as an individual class in a static analysis (aka holistic/component) ([6], [7], [15], [16], [17], [18], [19], [20], [21]). Paul Ekman and Wallace V. Friesen hypothesise these expressions are appropriate to be universally recognized ([22], [23]); and have devised a widely used system of metrics known as 'The Facial Action Coding System' (FACS) that captures facial actions that are applicable to facial expressions [24].

In order to define and evaluate expression classes of face images, a dataset is required. The JAFFE [7], Cohn-Kanade [25], FEEDTUM [26] and MMI [27] are common datasets which have been used in [12], ([8], [28], [29], [30], [31]),

Kenny Hong and Stephan K. Chalup are with the School of Electrical Engineering and Computer Science, The University of Newcastle, NSW, Australia (email: kenny.hong@newcastle.edu.au, stephan.chalup@newcastle.edu.au).

Robert A.R. King is with School of Mathematical and Physical Sciences, The University of Newcastle, NSW, Australia (email: robert.king@newcastle.edu.au).
([10], [32]), and ([8], [12]), respectively. To a lesser extent the Yale [33], AR [34], and SFED07 [35] datasets have been used in recent studies such as [11] and [20]—these studies use fewer expressions [11] and deviate from the typical [20]. Of the mentioned datasets only the Cohn-Kanade [25] and MMI [27] have cited certification of their face images using facial expression experts.

Once a dataset is acquired the decision of which preprocessing steps to utilise becomes a challenge due to the many variations available. Edge detection ([36], [37]) and histogram equalisation ([20], [21], [31]) are commonly used to counter the effects of noise from poor lighting. The method of Local Binary Patterns (LBP) has also become popular for tackling poor lighting and has shown promising results in facial expression classification ([12], [16]). Wavelet compression techiques have been used to reduce noise by taking into account the spatial information of the face. For instance, Haar-like (using the Integral Image [38]) and Gabor wavelets have been used in [30] and ([7], [16], [17], [18], [21]), respectively. Further studies involve the use of Active Appearance Modeling (AAM) [6] to describe the face using feature points ([11], [14], [39]). Labelled as a component approach feature points are relevant for action preprocessing.

Action preprocessing methods extract facial actions from a set of face images. An important factor to consider is how many and where the feature points are to be used for aligning and normalising the face images. For instance, ([12], [30], [40]) chose the eyes, [20] chose the eyes and middle of the nose, [11] chose the eyes and nose tip, [41] chose the eyes and lateral nose corners, [42] chose the eyes and philtrum, and [17] chose the eyes, nose and mouth.

Once the face images are normalised, methods such as difference imaging and optical flow fields are employed to extract facial actions. Difference imaging uses a neutral and an expressive face image which are subtracted from one another (aka holistic action) ([43], [44]) while optical flow fields compute the motion vector of pixels from an image sequence (aka component action) ([28], [45]); supplementary methods that have been used with facial actions include fuzzy logic and heuristics [28].

Our aim is to compare several image and action preprocessing steps using the discrete expressions: happy, sad, surprised, fearful, angry, disgusted and neutral; with a dataset aligned and normalised by our proposed face model. For the classification task we propose a modified multiclass support vector machine (SVM) that uses pairwise adaptive model parameters, and compare that to a standard multiclass SVM. Our aim is to find the best techniques and suggest a standard 
so that results can be compared. Our hypothesis is that a component based approach will outperform a holistic approach for facial expression analysis despite of ongoing interest in holistic approaches. An additional hypothesis is that by fine tuning the decision plane of a multiclass SVM via one adaptive parameter assignment per binary classifier, the performance of facial expression classification can be improved.

A system overview is presented in the next section, followed by a description of the dataset and preprocessing in section III and the classification of facial expressions in section IV. Section V presents the experimental results and discussion, and is followed by concluding remarks in Section VI.

\section{SYSTEM OVERVIEW}

The system consists of three preprocessing steps followed by the classification step. There are several preprocessing options. The full system structure is shown in Figure 1. The first preprocessing step is to align and normalise the face images in the dataset. Then, for comparison, the individual images are preprocessed to four image formatsgrayscale, LBP, edge images, and feature points. These are then organised into four prominent approaches-holistic, holistic action, component and component action. Details about further preprocessing and each module of the system are explained in subsequent sections.

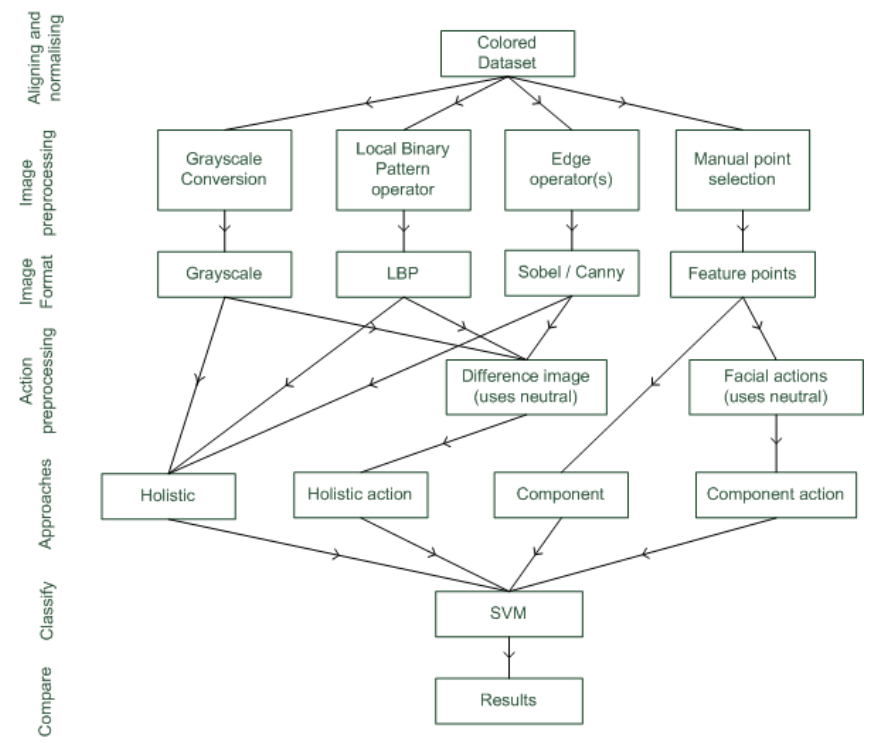

Fig. 1. System overview showing the flow of the stages and states.

\section{DATASET AND PREPROCESSING}

We generate our face images from the JACFEE and JACNEUF datasets [46] by Paul Ekman Group LLC which have been certified by facial expression experts. The combined dataset is composed of 260 coloured face images of $100 \times 100$ pixels, consisting of 140 neutrals and 20 images of 6 different facial expressions. For each face image 60 feature points were manually placed around the brows, eyes and mouth for use in face alignment and normalisation as shown in Figure 2. Each feature point is labelled as either:

1) Stable point (red colour): Stable points are invariant across facial expressions. They provide the basis for alignment and normalisation via affine transformations. We chose the inner eye corners (caruncles) as the stable points.

2) Active point (blue colour): Active points are the minimum set of feature points required to describe and distinguish between expressions. They exhibit high variance across facial expressions. We use the brow end points, mid-upper and mid-lower eyelids, lip end points, and mid-upper and mid-lower lips as the active points.

3) Passive point (green colour): Passive points are feature points to enhance a facial feature's shape and movement. They move in unison with the active points and are positioned between them. We have positioned several approximately equidistant passive points.

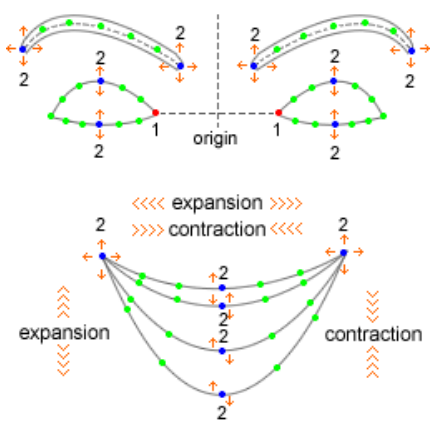

Fig. 2. Face model showing the feature points $[1=$ stable, $2=$ active, remainder are passive], facial actions and directions of horizontal and vertical expansion and contraction.

Affine transformations: The individual face images are aligned and normalised via translation, rotation and scaling such that the inner eye corners lie on the horizontal $x$-axis and the midpoint between the inner eye corners sits on the origin of the transformed plane. The transformation matrix is as follows:

$$
\begin{gathered}
\operatorname{normalize}(x, y)=\operatorname{scale}(x, y) \times \operatorname{rotate}(x, y) \times \operatorname{translate}(x, y) \\
\operatorname{translate}(x, y)=\left[\begin{array}{c}
-\frac{\mathrm{x}_{L}+\mathrm{x}_{R}}{2} \\
-\frac{\mathrm{y}_{L}+\mathrm{y}_{R}}{2}
\end{array}\right]\left[\begin{array}{l}
x \\
y
\end{array}\right]
\end{gathered}
$$

where $\mathrm{x}_{L}, \mathrm{x}_{R}, \mathrm{y}_{L}, \mathrm{y}_{R}$ are the left and right inner eye corners.

$$
\operatorname{rotate}(x, y)=\left[\begin{array}{cc}
\cos (-\theta) & -\sin (-\theta) \\
\sin (-\theta) & \cos (-\theta)
\end{array}\right]\left[\begin{array}{l}
x \\
y
\end{array}\right]
$$

where $\theta$ is the angle from the $x$-axis.

$$
\operatorname{scale}(x, y)=\frac{1}{2 \mathrm{x}_{R}}\left[\begin{array}{l}
x \\
y
\end{array}\right]
$$

where $\mathrm{x}_{R}$ is right eye corner. 


\section{A. Image preprocessing}

An ITU-R 601-2 luma transform was used to convert RGB (red green blue) colour into grayscale [47].

$$
\text { grayscale }=\text { red } * 0.299+\text { green } * 0.587+\text { blue } * 0.114
$$

A $3 \times 3$ neighborhood LBP operator was used [12]; and as LBP is a non-parametric transform no selection of parameters was required.

For the edge operators there are several parameters that impact the final edge image. Selection of the Sobel and Canny parameters was by visual evaluation for ideal face edges. The parameters and the ranges are as follows-lower threshold: 85 [0-255] and upper threshold: 170 [0-255] for Sobel and Canny; and blur: 0 [0-50] and gain: 5 [1-10] for Sobel only.

Examples of the grayscale, LBP, Sobel and Canny edges are shown in Figure 3. Further details are explained in the next subsection.

\section{B. Action preprocessing}

TABLE I

FACIAL ACTIONS ASSIGNED TO FEATURE POINTS IN THE FACE MODEL OF FIGURE 2.

\begin{tabular}{|l|l|}
\hline Feature points & Actions \\
\hline endpoints brow axis & left, right, up, down \\
midpoint of upper \& lower eyelid axis & up, down \\
left, right mouth corner points & up, down, left, right \\
midpoint of upper lip bottom axis & up, down \\
midpoint of lower lip top axis & up, down \\
upper lip bottom axis & horizontal contr./expansion \\
lower lip top axis & horizontal contr./expansion \\
midpoints of upper lip axes & vertical contr./expansion \\
midpoints of bottom lip axes & vertical contr./expansion \\
\hline
\end{tabular}

The facial actions assigned to feature points are shown in Table I and illustrated in Figure 2. Differences in the distance of feature points between a neutral and an expressive face image are used to determine the directional actions left, right, up and down. The horizontal contraction and expansion uses the difference of the sum of the distances between the feature points along the inner mouth opening (vermillion); while the vertical contraction and expansion uses the difference of the distance between the midpoints of the outer (vermillion border) and inner mouth opening (vermillion). The horizontal and vertical facial actions were introduced to describe the mouth more clearly as it has a higher degree of movement than the brows and eyes. Their equation is as follows:

$$
\operatorname{diff}=\sum_{i=0}^{n-1}\left(E_{i+1}-E_{i}\right)^{2}-\sum_{i=0}^{n-1}\left(N_{i+1}-N_{i}\right)^{2}
$$

where the $E_{i, i=0, \ldots, n-1}$ represent the expressive feature points, the $N_{i, i=0, \ldots, n-1}$ represent the neutral feature points, diff $>0$ is an expansion and $\operatorname{diff}<0$ is a contraction.

Figure 3 illustrates the combinations of image and action preprocessing steps used. The columns are examples of the preprocessed images with the rows showing the facial expressions and various attributes of the dataset. For instance, rows 2-4 are averaged images of the dataset showing clearly the inner eye corners that was used for alignment and normalisation. Rows 5-7 are averaged images of the differences between a neutral and expressive face - the scattered grainy effect suggests noise and it is more evident in the LBP and Canny edge formats than the grayscale and Sobel formats. Finally, rows 1-4 are face crop examples that are used for comparing with head crop examples of rows 5-7.

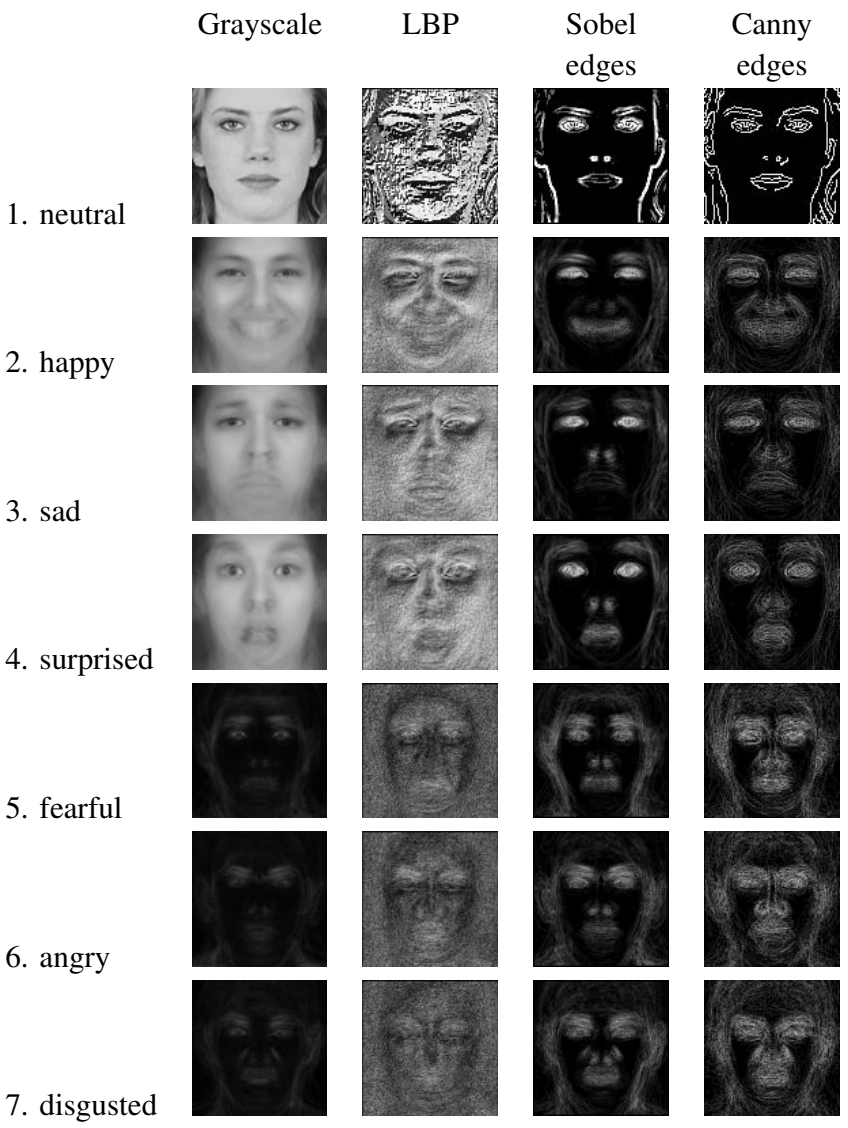

Fig. 3. Examples of expressions and preprocessing steps. The columns are the preprocessed images and the rows are different expressions. Specific rows show other attributes of the generated data such as the head crop in the averaged difference images of rows 5-7 compared to the face crop of rows 1 to 4 . The clarity of the inner eye corners of Rows 2 to 7 defines their use for alignment and normalisation. The data used to generate the above images was from Ekman's JACFEE and JACNEUF datasets [46].

\section{Classification of Facial EXPREsSions}

Support vector machines (SVM) was used in our study as they are one of the most effective tools for facial expression classification ([12], [14], [25], [30], [31]). Others have classified facial expressions using such methods as Neural Networks ([42], [48]) and k-Nearest Neighbors ([12], [49]). Dimensionality reduction methods have been used prior to classification such as principal component analysis (PCA) ([48], [49], [50], [51]), linear discriminant analysis (LDA) [52] and independent component analysis (ICA) ([31], [49]). Further, the concatenation of these methods has been tried and tested, for example PCA with NN [48], SVM with NN [42], PCA/ICA with kNN [49] and LDA with kNN [12].

In the subsequent subsections we provide a description of multiclass support vector machines followed by our proposed 
modified multiclass SVM using pairwise adaptive model parameters and the structure of the feature vectors used.

\section{A. Multiclass classification and kernel}

The basic description of support vector machines can be phrased as a two-class classification problem where datapoints are mapped into a high dimensional hyperspace so that they can be separated by a hyperplane [53]. A margin exists on each side of the hyperplane which is distanced to the nearest set of datapoints of each class. A high margin indicates good separation and good generalisation. The datapoints that sit on the margin are known as support vectors.

Current multiclass SVM approaches use two possible classification strategies: one-against-all and one-against-one. In a one-against-all approach, $q$ classes of SVMs are trained. The highest output value among the $q$ classes wins. In a one-against-one approach, a pairwise evaluation via a voting system in tree form is used to determine the overall winner. We use the one-against-one approach because of its suitability for application to a large dataset [54].

When using a SVM classifier there are three main kernels available: linear, polynomial and radial basis function (RBF). We chose the RBF kernel because several facial expression studies have reported higher results using RBF followed by the polynomial and then the linear kernel ([25], [12], [13]); while others have used the RBF ([8], [15], [11]), polynomial [10] and linear kernel [31] as their default choice. In fewer cases the polynomial ([30], [42]) and linear kernel [17] were reported to perform better than the RBF kernel.

The SVM with RBF kernel has two parameters to optimise: $C>0$ and $\gamma>0$ (Gamma). $C>0$ adjusts the penalty of the error term that maximises margin separation by minimising the primal form [53]:

$$
\min _{\mathbf{w}, b, \xi} \frac{1}{2} \mathbf{w}^{T} \mathbf{w}+C \sum_{i=1}^{l} \xi_{i}
$$

subject to

$$
y_{i}\left(\mathbf{w}^{T} \phi\left(\mathbf{x}_{i}\right)+b\right) \geq 1-\xi_{i},
$$

where $\mathbf{w}$ is the normal vector, $b$ is the bias, $\xi_{i} \geq 0$ are the slack variables, $\mathbf{x}_{i}$ are the samples and $\mathbf{y}_{i}$ are the labels $( \pm 1)$; while $\gamma>0$ adjusts the RBF kernel function:

$$
K\left(\mathbf{x}_{i}, \mathbf{x}_{j}\right)=\exp \left(-\gamma\left\|\mathbf{x}_{i}-\mathbf{x}_{j}\right\|^{2}\right), \gamma>0
$$

\section{B. Proposed pairwise adaptive Multiclass SVM}

We propose a pairwise multiclass SVM that uses adaptive model parameters to improve classification. What is different to the standard multiclass SVM is that individual $(C, \gamma)$ pairs are assigned to each binary classifier and their values are determined e.g. via a grid search. A voting strategy is then used to count each binary classifier of an expression. The maximum count gives the final expression. Equally weighted expressions are picked by the lowest index in the array [55]. The total number of binary classifiers required is $\left(\begin{array}{l}n \\ 2\end{array}\right)$ where $n$ is the number of expressions to evaluate. We use the
LIBSVM library for the classification task as the one-againstone multiclass strategy and grid search [55]. The grid search used in LIBSVM is a coarse to fine search that grows the sequences of $C$ and $\gamma$ exponentially.

\section{Feature vectors}

Four feature vectors were defined for each of the approaches by a sequence of values. The holistic and holistic action feature vectors consist of $100 \times 100$ dimensions of the image intensities; while the component feature vector consists of 120 dimensions of the 60 feature points, and the component action feature vector consists of 44 dimensions of the facial actions.

As the left, right, up, down actions are common to most of the feature points, it is of interest to know the accuracy using only these actions-this is a 36 dimensional feature vector which is the 44 dimensional feature vector without the horizontal and vertical expansions and contractions.

With the exception of the component approach all other feature vectors were normalised between -1 and +1 .

\section{RESUlts AND Discussion}

The general view is that classification improves when cropping closer to the facial features (i.e. face crop $>$ head crop) and excluding neutral. The component action based approach (using neutral) returned the best result with an accuracy of $98.33 \%$. This was followed by the component based approach with $80.83 \%$ accuracy (neutral excluded) and the holistic approach with an accuracy of $71.15 \%$ (grayscale and neutral excluded). Least accurate was the holistic action approach at $62.50 \%$ (grayscale and neutral excluded). The reported percentages are from the proposed pairwise adaptive multiclass SVM which performed favourably in many of the experiments conducted.

An overview of the results is shown in Table II. Details of the dataset, preprocessing steps, approaches and our proposed classifier are discussed further in subsequent subsections. In addition, we discuss our results in regards to the expression space prior to conclusion.

TABLE II

\begin{tabular}{|c|c|}
\hline Approach & Result \\
\hline Holistic & $\begin{array}{l}\text { Include neutral: } \\
\text { Grayscale > Sobel > } \\
\text { Canny } \geq \text { LBP } \\
\text { Exclude neutral: } \\
\text { LBP > Canny > } \\
\text { Grayscale > Sobel }\end{array}$ \\
\hline Holistic action & $\begin{array}{l}\text { Grayscale }>\text { LBP }> \\
\text { Canny }>\text { Sobel }\end{array}$ \\
\hline $\begin{array}{l}\text { Component } \\
\text { (feature pts.) }\end{array}$ & Exclude neutral $>$ include neutral \\
\hline $\begin{array}{l}\text { Component action } \\
\text { (facial actions) }\end{array}$ & $\begin{array}{l}\text { Include horizontal and vertical } \\
\text { expansion and contraction returns higher result }\end{array}$ \\
\hline Summary & $\begin{array}{l}\text { Image cropping: face crop }>\text { head crop } \\
\text { Neutral: exclude }>\text { include } \\
\text { Component action }>\text { component }> \\
\text { holistic }>\text { holistic action }\end{array}$ \\
\hline
\end{tabular}

OVERVIEW OF THE EXPERIMENTAL RESULTS: ORDER OF PERFORMANCE EVALUATED AS THE 10-FOLD CROSS VALIDATION SCORE. 


\section{A. Dataset}

Issues to be considered in the examination of our results include comparison with other relevant studies, the neutral expression, and whether our choice of pixel resolution was optimal.

To compare with other relevant studies we examined the best results of other facial classification studies that use the Cohn-Kanade and MMI datasets. For the Cohn-Kanade dataset, the studies which employed six expression classes (happy, sad, surprised, fearful, angry and disgusted) achieved 93.85\% [30] and 93.8\% [56]; and those with seven expression classes (the typical six plus neutral) achieved 93.8\% [17] and 93.3\% [13]. For the MMI dataset, the studies which employed six expression classes achieved $82.68 \%$ [8] and $92.6 \%$ [12]; and those with seven expression classes achieved $88.9 \%$ [12] and $93.61 \%$ [21]. In comparison, our best results employing six expression classes achieved $80.83 \%$ via a component approach, and for a seven expression class we achieved $98.33 \%$ via a component action approach.

The neutral face is an expression that is difficult to classify. One main reason is its subtle resemblance to other facial expressions which can be linked to the overgeneralization of an emotion recognition system [57]. To analyse this impact we use a standard two class RBF-SVM that compares the neutral expression with the rest of the expressions. A result of $88.85 \%$ (component approach using feature points) suggests that the SVM is capable of distinguishing between a neutral and an expressive face. This is supported by the Receiver Operator Characteristic Curve (ROC) shown in Figure 4 where the component approach (i.e. feature points) is the overall best performer. This is shown by the red dashed ROC curve with the maximum area under the curve.

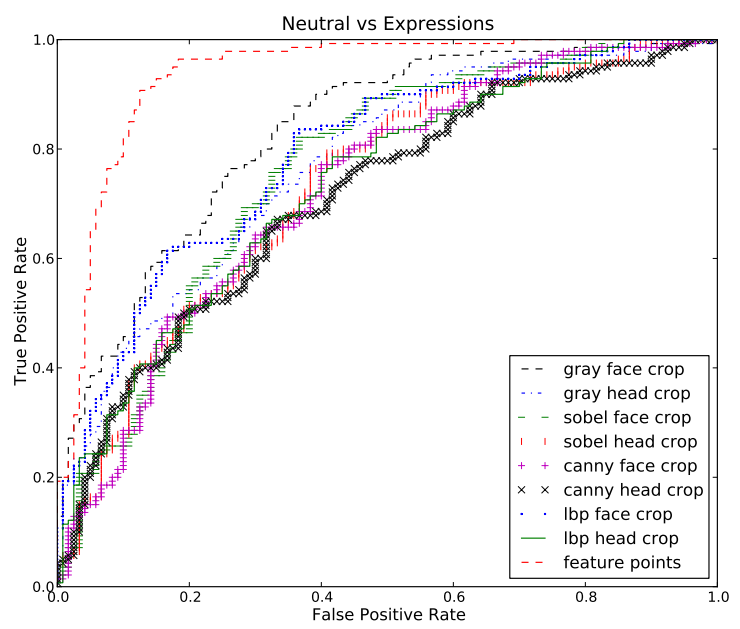

Fig. 4. ROC curves - Neutral vs. Expression. The component approach (i.e. feature points) is best performer for distinguishing between a neutral and an expressive face. This is shown by the red dashed ROC curve with the maximum area under the curve.

Was the generated data resolution optimal for our experiments? Several studies have experimented with multiple pixel resolutions to determine an optimal balance of image height and image width. For instance, [16] compared $64 \times 64$, $48 \times 48,32 \times 32$ and $16 \times 16$ on the JAFFE dataset, [12] compared $110 \times 150,55 \times 75,36 \times 48,27 \times 37,18 \times 24$ and $14 \times 19$ on the JAFFE, Cohn-Kanade and MMI dataset; and [56] compared $288 \times 384,144 \times 192,72 \times 96,36 \times 48$ and $18 \times 24$ on the Cohn-Kanade dataset. Each showed that the performance decreased when the face data were downsampled[16], [12], and [56] returned reduce performance when the pixel resolutions fell below $64 \times 64,110 \times 150$ and $72 \times 96$ respectively. We chose $100 \times 100$ pixels because it sits between $64 \times 64$ and $110 \times 150$ and the expressive features were visually clear.

\section{B. Image preprocessing}

The feature points of the component approach performed best - there was minimal noise when compared with other preprocessing steps and there were no parameters to tune; however the feature point had to be manually inserted. On the other hand, changing the edge operator's parameters in the holistic approach affects the visual clarity of the facial features in the final edge image. As there is currently no literature on determining the optimal parameters for edge filtering on face images our approach was to observe the face edge images as the upper and lower thresholds changed in ratios of 1:1, 1:2, 1:3 and so on. This gave an initial upper and lower threshold of 128 . When the thresholds are the same, specular edges appear. Because of this we moved to a 1:2 ratio of 85 and 170. After trials of other ratios we perceive that the 1:2 ratio was optimal for both the Sobel and Canny edge operators. In regards to the Sobel edge operator, we have left blur to 0 and a mid-gain of 5 as a good compromise between high and low attenuation of the edge images.

\section{Action preprocessing}

The most commonly used action preprocessing step in the literature is the 'Facial Action Coding System' (FACS) by Paul Ekman and W.V. Friesen [24]. The large list of 32 action units (AU) in [58] explains the many variations of feature points proposed. For instance, the brows, eyes and mouth are the primary areas studied, while some included the jawline ([8], [14], [59]), chin [32], forehead and between the eyes [15], and others included the cheeks [32] as well as wrinkles and furrows [9]. Of these variations, which feature points are relevant and which are redundant for detecting facial actions? We hypothesize that feature points that are easily detectable via image preprocessing are relevant and the remaining are redundant as they can be inferred by the relevant feature points. For instance, the action unit of raising cheek (AU 6) would be difficult to detect in image processing however can be deduced through detecting the action of raising the mouth corners and raising the lower eyelids. The wrinkling of the nose (AU 9) is another example. How does one separate transient wrinkles from permanent wrinkles (i.e. aging)? This can be difficult to detect in image processing however can be inferred by the raising of the upper lip and lowering of the inner brows. 


\section{Component or holistic approach}

There is still an on-going debate between the merits of the holistic and component approaches [5]. Our results indicate that a component based approach improves classification of discrete facial expressions over a holistic approach. Could it be the influence of holistic approaches from facial recognition? When face recognition research was first introduced, the primary methods relied mostly on feature points and distances between them [60]. This resulted in poor recognition and researchers looked into holistic methods that dealt with intensity values such as grayscale [61]. Facial expression studies emerged at the peak of the popularity of holistic methods [62] and soon a large set of literature on holistic approaches for facial expression analysis followed ([4], [5]).

\section{E. Pairwise adaptive multiclass SVM}

Our proposed pairwise adaptive multiclass SVM has shown better results than the standard multiclass SVM in many cases. Out of 28 experiments, only 8 reported a minor decrease in performance- 5 in holistic action, 2 in holistic and 1 in the component action approach. These results suggest that the proposed method is superior except for the case of the holistic action approach.

As the component action feature vectors are represented by binary strings, SVM multiclass classification may benefit from using Error Correcting Output Coding (ECOC) approaches [63].

\section{F. Optimal parameters for multiclass SVM}

Figure 5 plots the best $C, \gamma$ and correct classification rate of the standard multiclass SVM from the grid search. It clearly shows that the component action approach is ranked the highest followed by the component, holistic and holistic action approach. Figure 6 plots the best $C, \gamma$ and correct classification rate of the binary classifiers of our proposed pairwise multiclass SVM. The approaches are highlighted by colour coded circles and the largest circles represent the most frequent pair.

The question is if there is an optimal $C$ and $\gamma$ pair. This is not likely for the standard multiclass SVM as there was no repeated $(C, \gamma)$ pair. However for the pairwise adaptive multiclass SVM there were 26 times and 23 times repeated $(C, \gamma)$ pairs of $\log 2(-5,-7)$ with correct classification rates of $100 \%$ and $87.5 \%$, respectively. The 26 times repeated pair was composed of 9 component action feature vectors of 44 dimensions, 10 component action feature vectors of 36 dimensions, 6 component feature vectors and 1 holistic action feature vector in LBP format. The 23 times repeated pair was composed of 8 binary classifiers in Canny format, 2 in grayscale format, 2 in Sobel and 11 in LBP format.

As shown in Figure 7, there are several $C$ and $\gamma$ pairs that allow correct classification.

\section{G. Expression space}

Recently the topic of expression space based on discrete facial expressions was investigated ([18], [19]). In the experiments conducted, the performance of the pairwise expression

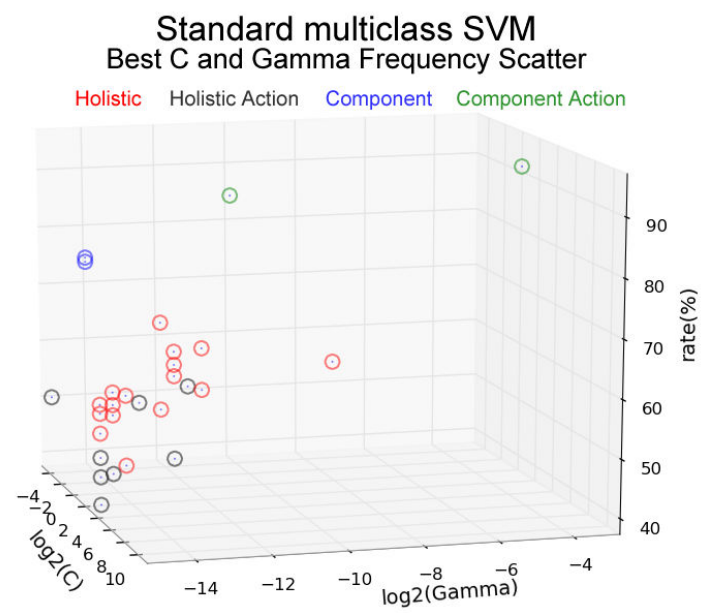

Fig. 5. This graph displays the correct classification rate of $(C, \gamma)$ pairs for all experiments from the standard multiclass SVM where the different approaches are indicated by colour coded circles.

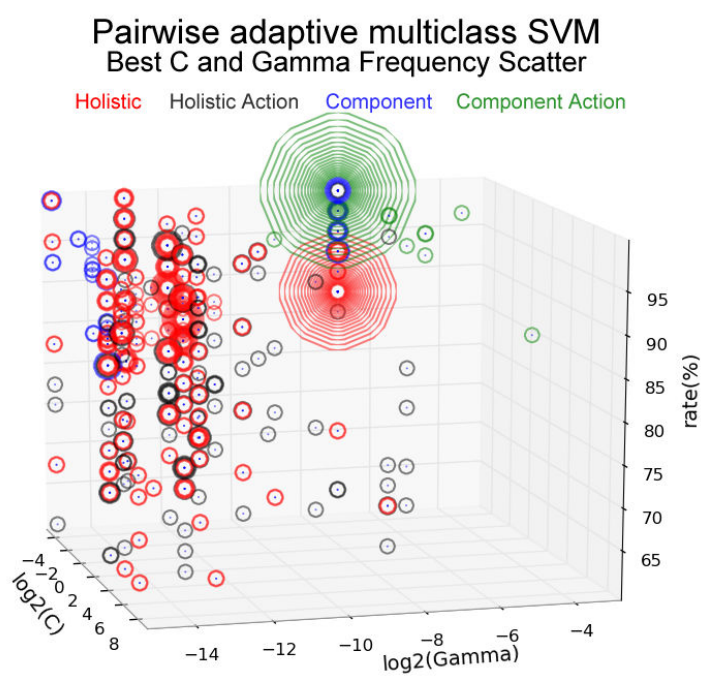

Fig. 6. In the pairwise adaptive multiclass SVM approach a $(C, \gamma)$ pairing can occur several times in one experiment. The frequency of $(C, \gamma)$ pairs is indicated by the number of increasing circles. The most frequently occuring pair is $\log 2(-5,-7)$ with a $100 \%$ correct classification rate.

pairs suggests that the weighting of the expressions are not equal. For instance, the fearful and surprised pair from Table III performed poorly while the happy and surprised pair gave the best result. The poor performance of the fearful and surprised pair corresponds to the difficulty in distinguishing the two expressions that is apparent in preliterate cultures [64] — suggesting that the expression space of fearful and surprised has a larger overlapped region than that of happy and surprised.

Further, these results agree with studies such as [14] which showed that participants performed poorly at separating anger and disgust while happy and surprised were easily distinguished. That study excluded sadness and fear as they decided that these expressions were not as distinctive as the other basic emotions. Our fearful-sadness expression pair from Table III agrees with their conclusion as it is ranked 
Component action - C and Gamma Grid Search

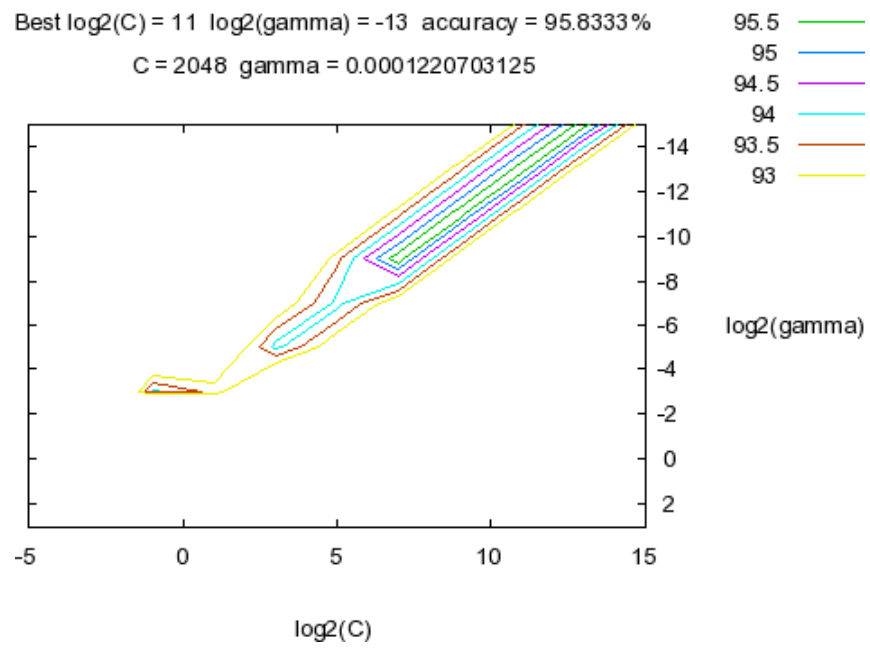

Fig. 7. Example grid search of the component action approach which shows that $C$ and $\gamma$ are not unique to the classification rate.

third last.

TABLE III

EXPRESSION PAIRS - AVERAGED 10 FOLD CROSS VALIDATION SCORE FOR THE CORRESPONDING PAIRWISE CLASSIFICATION

\begin{tabular}{|l|l|l|l|l|l|}
\hline Pair & Rate & Pair & Rate & Pair & Rate \\
\hline hap,sur & 96.88 & fea,neu & 90.69 & ang,sad & 85.09 \\
hap,fea & 93.75 & ang,neu & 90.25 & dis,sad & 84.11 \\
sur,ang & 93.04 & hap,sad & 89.82 & sur,sad & 82.86 \\
sur,neu & 92.13 & dis,fea & 88.84 & hap,dis & 80.45 \\
hap,neu & 91.94 & sad,neu & 87.88 & sad,fea & 76.69 \\
dis,neu & 91.06 & ang,fea & 87.59 & ang,dis & 74.20 \\
sur,dis & 90.98 & hap,ang & 87.05 & fea,sur & 74.11 \\
\hline
\end{tabular}

\section{CONCLUSION}

The current approaches to facial expression classification employ a variety of expression classes and different preprocessing steps, which makes comparison of results difficult. This study was initiated to find the best techniques and suggest a standard so that results can be compared. From our literature review we found that there was a lack of certified datasets publicly available and many did not cover the neutral expression. Our comparison showed that including the neutral expression leads to decreased performance in classifying the discrete expressions. Reported classification rates will be artificially higher for studies that exclude the neutral expression. A face model was proposed for aligning and normalising the datasets. The roles of each of the feature points in the face model were justified-this was not clear in the literature. For the classification task, our proposed pairwise adaptive multiclass SVM performed better than the standard multiclass SVM in many of the experiments conducted except for the holistic action approach, which involved difference imaging of face images. Further, the performance of the binary classifiers in our proposed method supports the unequal weighting of the expression space as we observed specific pairs of expressions performing better than others, and these observations correspond to psychological studies using real human subjects.

\section{ACKNOWLEDGMENT}

This work was supported in part by the Australian Research Council Discovery Project DP0770106.

\section{REFERENCES}

[1] P. Ekman, Emotions Revealed. Henry Holt and Company 115 West 18th Street New York, New York 10011: Times Books, 2003.

[2] A. Jaimes and N. Sebe, "Multimodal human-computer interaction: A survey," Computer Vision and Image Understanding, vol. 108, no. 12, pp. 116-134, 2007, Special Issue on Vision for Human-Computer Interaction.

[3] Hjelmas E. and Low B.K., "Face detection: A survey," Computer Vision and Image Understanding, vol. 83, no. 39, pp. 236-274, 2001.

[4] B. Fasel and J. Luettin, "Automatic facial expression analysis: A survey," Pattern Recognition, vol. 36, no. 1, pp. 259-275, 2003.

[5] Z. Zeng, M. Pantic, G. I. Roisman, and T. S. Huang, "A survey of affect recognition methods: Audio, visual, and spontaneous expressions," IEEE Transactions on Pattern Analysis and Machine Intelligence, vol. 31, no. 1, pp. 39-58, 2009.

[6] A. Lanitis, C. J. Taylor, and T. F. Cootes, "Automatic interpretation and coding of face images using flexible models," IEEE Transactions on Pattern Analysis and Machine Intelligence, vol. 19, no. 7, pp. 743756, 1997.

[7] M. J. Lyons, S. Akamatsu, M. Kamachi, and J. Gyoba, "Coding facial expressions with gabor wavelets," in Proceedings of Third IEEE International Conference on Automatic Face and Gesture Recognition, 1998, pp. 200-205.

[8] J. Wang and L. Yin, "Static topographic modeling for facial expression recognition and analysis," Computer Vision and Image Understanding, vol. 108, no. 1-2, pp. 19-34, 2007, Special Issue on Vision for HumanComputer Interaction.

[9] Y. Zhang and Q. Ji, "Active and dynamic information fusion for facial expression understanding from image sequences," IEEE Transactions on Pattern Analysis and Machine Intelligence, vol. 27, no. 5, pp. 699714, 2005.

[10] F. Wallhoff, B. Schuller, M. Hawellek, and G. Rigoll, "Efficient recognition of authentic dynamic facial expressions on the feedtum database," 2006 IEEE International Conference on Multimedia and Expo, pp. 493-496, 2006.

[11] S. Park and D. Kim, "Subtle facial expression recognition using motion magnification," Pattern Recognition Letters, vol. 30, no. 7, pp. 708716, 2009.

[12] C. Shan, S. Gong, and P. W. McOwan, "Facial expression recognition based on local binary patterns: A comprehensive study," Image and Vision Computing, vol. 27, no. 6, pp. 803-816, 2009.

[13] M. Bartlett, G. Littlewort, M. Frank, C. Lainscsek, I. Fasel, and J. Movellan, "Recognizing facial expression: machine learning and application to spontaneous behavior," in Computer Vision and Pattern Recognition, 2005. CVPR 2005. IEEE Computer Society Conference on, vol. 2, June 2005, pp. 568-573.

[14] M. Beszedes and P. Culverhouse, "Comparison of human and automatic facial emotions and emotion intensity levels recognition," 5th International Symposium on Image and Signal Processing and Analysis, pp. 429-434, 2007.

[15] G. Guo and C. Dyer, "Learning from examples in the small sample case: face expression recognition," IEEE Transactions on Systems, Man, and Cybernetics, Part B, vol. 35, no. 3, pp. 477-488, 2005.

[16] S. Liao, W. Fan, A. Chung, and D.-Y. Yeung, "Facial expression recognition using advanced local binary patterns, tsallis entropies and global appearance features," in IEEE International Conference on Image Processing, Oct. 2006, pp. 665-668.

[17] G. Littlewort, M. S. Bartlett, I. Fasel, J. Susskind, and J. Movellan, "Dynamics of facial expression extracted automatically from video," Image and Vision Computing, vol. 24, no. 6, pp. 615-625, 2006.

[18] W. Liu, J. Lu, Z. Wang, and H. Song, "An expression space model for facial expression analysis," in Congress on Image and Signal Processing, 2008. CISP '08, vol. 2, May 2008, pp. 680-684.

[19] H. Wang and K. Wang, "Affective interaction based on personindependent facial expression space," Neurocomputing, vol. 71, no. 10-12, pp. 1889-1901, 2008, Neurocomputing for Vision Research; Advances in Blind Signal Processing. 
[20] X. Xie and K.-M. Lam, "Facial expression recognition based on shape and texture," Pattern Recognition, vol. 42, no. 5, pp. 1003-1011, 2009.

[21] M. Kyperountas, A. Tefas, and I. Pitas, "Salient feature and reliable classifier selection for facial expression classification," Pattern Recognition, vol. 43, no. 3, pp. 972-986, 2010.

[22] P. Ekman and W. Friesen, "Constants across cultures in the face and emotion." Journal of Personality and Social Psychology, vol. 17, no. 2, pp. 124-129, 1971.

[23] P. Ekman and W. V. Friesen, "A new pan-cultural facial expression of emotion," Motivation and Emotion, vol. 10, no. 2, pp. 159-168, 1986.

[24] P. Ekman and W. Friesen, Facial Action Coding System: A Technique for the Measurement of Facial Movement. Consulting Psychologists Press, 1978.

[25] T. Kanade, J. Cohn, and Y. Tian, "Comprehensive database for facial expression analysis," in Proceedings of Fourth IEEE International Conference on Automatic Face and Gesture Recognition, 2000, pp. $46-53$.

[26] F. Wallhoff. (2005) The facial expressions and emotions database homepage (feedtum). [Online]. Available: www.mmk.ei.tum.de/waf/ fgnet/feedtum.html

[27] M. Pantic, M. Valstar, R. Rademaker, and L. Maat, "Web-based database for facial expression analysis," in IEEE International Conference on Multimedia and Expo, July 2005.

[28] Y. Tian, T. Kanade, and J. F. Cohn, "Recognizing action units for facial expression analysis," IEEE Transactions on Pattern Analysis and Machine Intelligence, vol. 23, no. 2, pp. 97-115, Feb 2001.

[29] R. Zhi and Q. Ruan, "Discriminant spectral analysis for facial expression recognition," in 15th IEEE International Conference on Image Processing, Oct. 2008, pp. 1924-1927.

[30] G. Zhao and M. Pietikinen, "Boosted multi-resolution spatiotemporal descriptors for facial expression recognition," Pattern Recognition Letters, vol. 30, no. 12, pp. 1117-1127, 2009.

[31] C.-F. Chuang and F. Y. Shih, "Recognizing facial action units using independent component analysis and support vector machine," Pattern Recognition, vol. 39, no. 9, pp. 1795-1798, 2006.

[32] A. Khanum, M. Mufti, M. Y. Javed, and M. Z. Shafiq, "Fuzzy case-based reasoning for facial expression recognition," Fuzzy Sets and Systems, vol. 160, no. 2, pp. 231-250, 2009, contribution of Fuzziness \& Uncertainty to Modern Artificial Intelligence, Case-Based Reasoning.

[33] A. Georghiades, P. Belhumeur, and D. Kriegman, "From few to many: Illumination cone models for face recognition under variable lighting and pose," IEEE Transactions on Pattern Analysis and Machine Intelligence, vol. 23, no. 6, pp. 643-660, 2001.

[34] A. Martinez and R. Benavente, "The AR Face Database," MIT Computer Vision Center 24, Tech. Rep., 1998.

[35] S. Park, H.-S. Lee, J. Shin, and D. Kim, "The POSTECH subtle facial expression database 2007(SFED07)," in 8th POSTECH-KYUTECH Joint Workshop On Neuroinformatics, 2008, pp. 653-654.

[36] R. Contreras, O. Starostenko, and L. Pulido, "An improved method for facial features extraction in images," in Electronics, Robotics and Automotive Mechanics Conference. CERMA '08, Oct 2008, pp. 261266.

[37] X. Qiu, W. Wang, J. Song, X. Zhang, and S. Liu, "Face recognition based on binary edge map and support vector machine," in The 1st International Conference on Bioinformatics and Biomedical Engineering, 2007, pp. 519-522.

[38] P. Viola and M. Jones, "Robust real-time face detection," International Journal of Computer Vision, vol. 57, no. 2, pp. 137-154, 2004.

[39] G. J. Edwards, T. F. Cootes, and C. J. Taylor, "Face recognition using active appearance models," pp. 581-595, 1998.

[40] M. Bartlett, G. Littlewort, M. Frank, C. Lainscsek, I. Fasel, and J. Movellan, "Fully automatic facial action recognition in spontaneous behavior," in 7th International Conference on Automatic Face and Gesture Recognition, 2006, pp. 223-230.

[41] Y. Tong, Y. Wang, Z. Zhu, and Q. Ji, "Robust facial feature tracking under varying face pose and facial expression," Pattern Recognition, vol. 40, no. 11, pp. 3195-3208, 2007.

[42] K. K. Lee and Y. Xu, "Real-time estimation of facial expression intensity," in Proceedings of IEEE International Conference on Robotics and Automation, vol. 2, 2003, pp. 2567-2572.

[43] M. S. Bartlett, "Face image analysis by unsupervised learning and redundancy reduction," Ph.D. dissertation, University of California, San Diego, 1998.
[44] G. Donato, M. Bartlett, J. Hager, P. Ekman, and T. Sejnowski, "Classifying facial actions," IEEE Transactions on Pattern Analysis and Machine Intelligence, vol. 21, no. 10, pp. 974-989, Oct 1999.

[45] M. Yeasin, B. Bullot, and R. Sharma, "Recognition of facial expressions and measurement of levels of interest from video," IEEE Transactions on Multimedia, vol. 8, no. 3, pp. 500-508, June 2006.

[46] Paul Ekman Group LLC. JACFEE and JACNEUF dataset. [Online]. Available: http://www.davidmatsumoto.com/research.php

[47] Studio Encoding Parameters of Digital Television for Standard 4:3 and Wide-Screen 16:9 Aspect Ratios, International Telecommunication Union Std., 2007.

[48] M. Bartlett, J. Hager, P. Ekman, and T. Sejnowski, "Measuring facial expressions by computer image analysis," Psychophysiology, vol. 36 , no. 2, pp. 253-263, 1999.

[49] B. Fasel and J. Luettin, "Recognition of asymmetric facial action unit activities and intensities," in Proceedings of 15th International Conference on Pattern Recognition, vol. 1, 2000, pp. 1100-1103.

[50] W. O. Odoyo, Geum-Boon Lee, Jung-Jin Park, and Beom-Joon Cho, "Facial expression classification using eigen-components of principal expressions," in 11th International Conference on Advanced Coтmunication Technology, vol. 3, Feb. 2009, pp. 1903-1906.

[51] M. Amin and H. Yan, "Expression intensity measurement from facial images by self organizing maps," in International Conference on Machine Learning and Cybernetics, vol. 6, 2008, pp. 3490-3496.

[52] M. J. Lyons, J. Budynek, and S. Akamatsu, "Automatic classification of single facial images," IEEE Transactions on Pattern Analysis and Machine Intelligence, vol. 21, no. 12, pp. 1357-1362, 1999.

[53] V. N. Vapnik, Statistical learning theory. John Wiley \& Sons, New York, 1998.

[54] Chih-wei Hsu and Chih-Jen Lin, "A comparison of methods for multi-class support vector machines," IEEE Transactions on Neural Networks, vol. 13, no. 2, pp. 415-425, 2002.

[55] Chih-Chung Chang and Chih-Jen Lin. (2001, June) Libsvm: a library for support vector machines. [Online]. Available: http: //www.csie.ntu.edu.tw/ cjlin/libsvm/

[56] Y. li Tian, "Evaluation of face resolution for expression analysis," in Conference on Computer Vision and Pattern Recognition Workshop CVPRW' 04 , June 2004, pp. 82-82.

[57] C. P. Said, N. Sebe, and A. Todorov, "Structural resemblance to emotional expressions predicts evaluation of emotionally neutral faces," Emotion, vol. 9, no. 2, pp. 260-264, 2009.

[58] P. Ekman, W. Friesen, and J. Hager, Facial Action Coding System Manual, 2002.

[59] J. Reilly and J. McDonald, "Modelling the manifold of facial expression using texture," in International Machine Vision and Image Processing Conference, 2008, pp. 63-68.

[60] A. Samal and P. A. Iyengar, "Automatic recognition and analysis of human faces and facial expressions: a survey," Pattern Recognition, vol. 25, no. 1, pp. 65-77, 1992.

[61] W. Barrett, "A survey of face recognition algorithms and testing results," in Conference Record of the Thirty-First Asilomar Conference on Signals, Systems \& Computers, vol. 1, Nov 1997, pp. 301-305.

[62] S. Kaiser and T. Wehrle, "Automated coding of facial behavior in human-computer interactions with facs," Journal of Nonverbal Behavior, vol. 16 , no. 2 , pp. $67-83,1992$

[63] F. F. Masulli and G. Valentini, "Effectiveness of error correction output coding decomposition schemes in ensemble and monolithic learning machines," Pattern Analysis and Application Journal, vol. 6, no. 4 , pp. $285-300,2003$.

[64] P. Ekman, Handbook of Cognition and Emotion. New York: John Wiley and Sons Ltd, 1999, ch. 16, pp. 301-320. 